\title{
Asymptotic Analysis of Cross-Hole Hydraulic Tests in Fractured Granite
}

\author{
by Walter A. IIIman ${ }^{1,2}$ and Daniel M. Tartakovsky ${ }^{3,4}$
}

\begin{abstract}
Illman and Tartakovsky (2005a, 2005b) developed a new approach for the interpretation of three-dimensional pneumatic well tests conducted in porous or fractured geologic media, which is based on a straight-line analysis of late-time data. We modify this approach to interpret three-dimensional well tests in the saturated zone and use it to analyze the cross-hole hydraulic test data collected in the Full-Scale Engineered Barrier Experiment gallery at the Grimsel Test Site in Switzerland. The equivalent hydraulic conductivity and specific storage obtained from our analysis increase with the radial distance between the centroids of the pumping and monitoring intervals. Since this scale effect is observed from a single test type (cross-hole tests), it is less ambiguous than scale effects typically inferred from laboratory and multiple types of hydraulic tests (e.g., slug, single- and cross-hole tests). The statistical analysis of the estimated hydraulic parameters shows a strong correlation between equivalent hydraulic conductivity and specific storage.
\end{abstract}

\section{Introduction}

Well test analyses in porous and fractured geologic media continue to be a topic of great importance because the parameters estimated from such tests have a direct bearing on the field-scale prediction of ground water flow and contaminant transport. There are several analytic techniques based on steady-state and transient analysis of drawdown data.

Arguably, the most widely used approach relies on type curves to interpret single- and cross-hole hydraulic and pneumatic test data in fractured rocks. Of direct relevance to the present analysis are the type-curve approaches of Hsieh and Neuman (1985) — who developed a fully three-dimensional analytic solution for flow to a well in an

${ }^{1}$ Corresponding author: Department of Geoscience, University of lowa, lowa City, IA 52242; (319) 335-1827; fax (319) 3351821; walter-illman@uiowa.edu

2Department of Civil \&t Environmental Engineering, and IIHRHydroscience \&t Engineering, University of lowa, lowa City, IA 52242.

${ }^{3}$ Department of Mechanical and Aerospace Engineering, University of California, San Diego, La Jolla, CA 92093; (858) 5341375; fax (858) 534-7599; dmt@ucsd.edu

${ }^{4}$ Theoretical Division, Los Alamos National Laboratory, Los Alamos, NM 87545.

Received August 2005, accepted December 2005.

Copyright (c) 2006 The Author(s)

Journal compilation (c) 2006 National Ground Water Association.

doi: 10.1111/j.1745-6584.2006.00201.x anisotropic medium that accounts for variable length injection and monitoring intervals as well as their angular dependence — and of Illman and Neuman (2001) — who modified this solution to account for airflow conditions as well as observation well storage and skin effects. Despite their popularity, type curves can be difficult to construct and use, especially if they are not implemented in commercially available software. More critically, many data sets cannot be analyzed with type curves because external forcings, small pressure responses, early-time nonlinear effects, and rock heterogeneity alter the pressure transients and preclude a good fit between the data and the type curves (e.g., Illman and Neuman 2000, 2001).

These problems can often be overcome by using only the steady-state portion of pressure-transient curves (e.g., Illman and Neuman 2003). A major advantage of steady-state analyses is that they do not require type curves and that estimates of equivalent permeabilities can be obtained rapidly if reliable steady-state pressure records are available. However, in many cases, drawdown or pressure-transient records do not reach a steady-state regime because pumping tests are not run long enough for drawdowns to reach a steady state. In addition, in oneand two-dimensional flow fields, a steady-state regime never develops unless the drawdown reaches a constanthead boundary or there is sufficient leakage from over and/ or underlying formations. If and when this occurs, the 
resulting drawdown is affected by boundary effects, which are not incorporated in many type-curve models. Finally, steady-state approaches do not yield estimates of porosity.

By using intermediate to late-time transient data, the asymptotic method of Illman and Tartakovsky (2005a, 2005b) overcomes many weaknesses of the existing steady-state and transient approaches. The method is based on an asymptotic approximation of the point-injection/point-observation, point-injection/line-observation, and the line-injection/line-observation solutions, which results in pressure varying linearly with $t^{-1 / 2}$. It merely requires plotting the data on a pressure vs. the reciprocal of the square root of time, which causes the pressure transient to develop a straight-line regime. The advantages of the asymptotic method include the following.

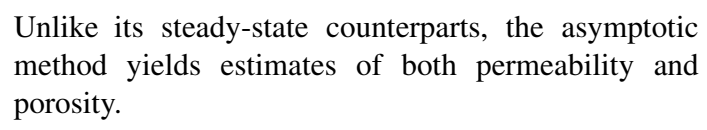
method yields estimates of both permeability and porosity.

Unlike its transient counterparts, the asymptotic analysis does not require the construction of type curves and, hence, is much easier to conduct. It eliminates the fitting of data to type curves, a procedure that requires considerable experience by the hydrogeologist and is highly subjective.

Unlike numerical inverse analyses, the asymptotic analysis does not require significant computer power and, hence, can be applied to any number of singleand cross-hole tests.

The asymptotic method works well for pressure records that exhibit a straight-line behavior, even if the signal-to-noise ratio is too low to allow meaningful steady-state and transient analyses. This includes cases where pressure transients are heavily affected by borehole storage, external forcings, and heterogeneities that cause the data to depart from analytically derived type-curve models.

Asymptotic solutions are currently available for pneumatic well tests in which air is injected into unsaturated porous and fractured formations (Illman and Tartakovsky, 2005a, 2005b). Here, we adopt these solutions to interpret cross-hole hydraulic tests and apply them to analyze data from fractured granite in the Grimsel Test Site (GTS) in Switzerland. The hydraulic conductivity estimates derived from our asymptotic analysis are compared with other site estimates including those determined by pulse, single-hole recovery, and cross-hole hydraulic tests as well as those determined from tunnel inflow measurements. We then examine the relationship between these two equivalent hydraulic parameters and radial distance between the centroids of the injection and monitoring intervals. The radial distance nominally represents the measurement scale of the equivalent hydraulic parameters. Finally, we examine the correlation between the equivalent hydraulic parameters.

\section{Asymptotic Analysis of Cross-Hole Hydraulic Tests}

For purposes of analyzing cross-hole hydraulic tests in fractured rocks, we follow Hsieh and Neuman (1985) to represent the rock by an infinite, three-dimensional, uniform, but anisotropic continuum that is characterized by hydraulic conductivity tensor $\mathbf{K}$ and specific storage $S_{\mathrm{s}}$. The injection and observation intervals are idealized, depending on their lengths, as points or lines. In the following analysis, we deal only with fluid injection into the test interval. The analysis is identical for fluid withdrawal if the increase in hydraulic head is replaced by decrease in hydraulic head.

The following four cases are considered: (1) injection at a point, observation at a point; (2) injection along a line, observation at a point; (3) injection at point, observation along a line; and (4) injection along a line, observation along a line. Figure 1 shows the geometric configuration of injection and observation intervals for all four test types in the working coordinate system.

The corresponding analytic solutions for drawdown $s$ in the monitoring well range from a relatively simple expression for the point-injection/point-observation test configuration (equation 4 of Hsieh and Neuman 1985) to progressively more evolved expressions for the lineinjection/point-observation, point-injection/line-observation, and line-injection/line-observation test configurations, given by equations 17, 27, and 35 of Hsieh and Neuman (1985), respectively.

A key to the asymptotic method of Illman and Tartakovsky $(2005 a, 2005 b)$ is the observation that, for large times $t$, all these solutions can be approximated by a simple expression

$$
s(t)=A-B t^{-1 / 2}
$$

with the coefficients $A$ and $B$ given subsequently for each test configuration. The linear behavior of drawdown $s$

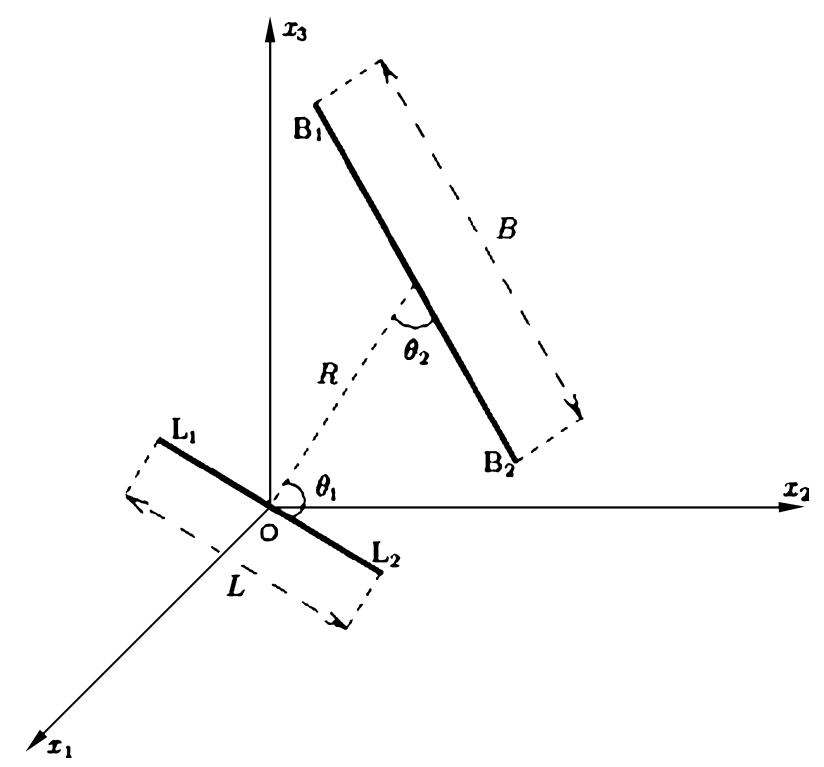

Figure 1. A schematic representation of a cross-hole injection test. The lengths of injection $\left(L_{1} L_{2}\right)$ and monitoring $\left(B_{1} B_{2}\right)$ intervals are $L$ and $B$, respectively. The centroid of the former coincides with the origin of the coordinate system, while the centroid of the latter is located at the distance $R$ away. The angles between the line connecting the two centroids and the injection and monitoring intervals are $\theta_{1}$ and $\theta_{2}$, respectively. 
with $t^{-1 / 2}$ leads to the following straight-line data interpretation procedure.

The drawdown data from a given monitoring interval are plotted against $t^{-1 / 2}$, the reciprocal of the square root of time. A straight line should develop for a portion of the data, to which a straight line is fitted. Let $s^{*}$ and $t^{*}$ denote the drawdown and time at which this straight line intersects the $t^{-1 / 2}$ axis and the $s$ axis, respectively. Then, Equation 1 yields

$$
A=s^{*}, \quad B=s^{*} \sqrt{t^{*}}
$$

from which hydraulic conductivity and specific storage can be readily estimated.

\section{Point Injection/Point Observation}

The coefficients $A$ and $B$ in Equation 1 are given by (Illman and Tartakovsky 2005a)

$$
A=\frac{Q}{4 \pi R} \sqrt{\frac{K_{\mathrm{d}}(\mathbf{e})}{D}}, \quad B=\frac{Q}{4 \pi^{3 / 2}} \sqrt{\frac{S_{s}}{D}}
$$

where $R$ is the distance between the injection and monitoring points (which can be thought of as the centroids of injection and monitoring intervals whose respective length $L, B \rightarrow 0$, as shown in Figure 1), $Q$ is flow rate, $D$ and $K_{\mathrm{d}}$ are the determinant and the canonical ellipsoids of $\mathbf{K}$, and

$$
K_{\mathrm{d}}(\mathbf{e})=\frac{1}{\mathbf{e}^{T} \mathbf{K}^{-1} \mathbf{e}}
$$

is the canonical ellipsoid of $\mathbf{K}$, with $\mathbf{e}$ being the unit vector along the line connecting the injection and observation points.

Then Equation 3 yields

$$
\sqrt{\frac{D}{K_{\mathrm{d}}(\mathbf{e})}}=\frac{Q}{4 \pi R s^{*}}, \quad S_{s}=\frac{\pi K_{d}(\mathbf{e}) t^{*}}{R^{2}}
$$

from which specific storage and the principal components of the conductivity tensor and the corresponding canonical ellipse $K_{\mathrm{d}}$ are determined following the procedure outlined by Hsieh et al. (1985).

For isotropic media, $\mathbf{K}=K \mathbf{I}$, where $\mathbf{I}$ is the identity matrix, and Equation 5 simplifies to give the following expressions for hydraulic conductivity and specific storage

$$
K=\frac{Q}{4 \pi R s^{*}}, \quad S_{s}=\frac{Q t^{*}}{4 R^{3} s^{*}}
$$

\section{Line Injection/Point Observation}

The coefficients $A$ and $B$ in Equation 1 are given by Illman and Tartakovsky (2005b):

$$
A=\frac{Q}{4 \pi L} \sqrt{\frac{K_{\mathrm{d}}\left(\mathbf{e}_{1}\right)}{D}} s_{\mathrm{d}_{\mathrm{LP}}}(\infty), \quad B=\frac{Q R}{4 \pi^{3 / 2} L} \sqrt{\frac{K_{\mathrm{d}}\left(\mathbf{e}_{1}\right) S_{s}}{K_{\mathrm{d}}(\mathbf{e}) D}}
$$

where $s_{\mathrm{d}_{\mathrm{LP}}}(\infty)$ is given by equation 52 of Hsieh and Neuman (1985),

$$
s_{\mathrm{LP}}(\infty)=\ln \frac{\sqrt{\alpha_{1}^{2}+2 \alpha_{1} \alpha_{2}+1}+\alpha_{1} \alpha_{2}+1}{\sqrt{\alpha_{1}^{2}-2 \alpha_{1} \alpha_{2}+1}+\alpha_{1} \alpha_{2}-1}
$$

$L$ is the injection interval length, $\mathbf{e}_{1}$ is the unit vector in the direction of the injection interval, and parameters $\alpha_{1}$ $\geq 0$ and $0 \leq \alpha_{2} \leq 1$, describing the geometric relationships between the injection and monitoring intervals, can be found in Hsieh and Neuman (1985). For isotropic media, $\alpha_{1}=2 R / L$ and $\alpha_{2}=\cos \theta_{1}$, where $\theta_{1}$ is the angle between the injection interval and the line connecting the centroids of the injection and monitoring intervals (Figure 1).

Then, Equation 7 gives the following expressions to infer the hydraulic conductivity and specific storage of an anisotropic formation,

$$
\sqrt{\frac{D}{K_{\mathrm{d}}\left(\mathbf{e}_{l}\right)}}=\frac{Q s_{\mathrm{d}_{\mathrm{LP}}}(\infty)}{4 \pi L s^{*}}, \quad S_{s}=\frac{\pi K_{\mathrm{d}}(\mathbf{e}) t^{*} s_{\mathrm{d}_{\mathrm{LP}}}^{2}(\infty)}{R^{2}}
$$

For isotropic formations, these reduce to

$$
K=\frac{Q s_{\mathrm{d}_{\mathrm{LP}}}^{2}(\infty)}{4 \pi L s^{*}}, \quad S_{s}=\frac{\pi K t^{*} s_{\mathrm{d}_{\mathrm{LP}}}^{2}(\infty)}{R^{2}}
$$

This approach should be used for data collected at

$$
t_{\mathrm{d}} \gg \frac{1-\alpha_{2}^{2}}{4}, \quad t_{\mathrm{d}} \gg \frac{\left(\alpha_{2} \pm 1 / \alpha_{1}\right)^{2}}{4}, \quad t_{\mathrm{d}}=\frac{K_{\mathrm{d}}(\mathbf{e}) t}{R^{2} S_{s}}
$$

For isotropic formations, $t_{\mathrm{d}}=K t / S_{\mathrm{s}} R^{2}$.

\section{Point Injection/Line Observation}

The coefficients $A$ and $B$ in Equation 1 are given by Illman and Tartakovsky (2005b):

$$
A=\frac{Q s_{\mathrm{d}_{\mathrm{PL}}}(\infty)}{4 \pi R} \sqrt{\frac{K_{\mathrm{d}}(\mathbf{e})}{D}}, \quad B=\frac{Q}{4 \pi^{3 / 2}} \sqrt{\frac{S_{s}}{D}}
$$

where $s_{\mathrm{dPL}}(\infty)$ is given by equation 53 of Hsieh and Neuman (1985),

$$
s_{\mathrm{dPL}}(\infty)=\frac{\beta_{1}}{2} \ln \frac{\sqrt{\beta_{1}^{2}+2 \beta_{1} \beta_{2}+1}+\beta_{1} \beta_{2}+1}{\sqrt{\beta_{1}^{2}-2 \beta_{1} \beta_{2}+1}+\beta_{1} \beta_{2}-1}
$$

and parameters $\beta_{1} \geq 0$ and $0 \leq \beta_{2} \leq 1$ describe the geometric relationships between the injection and monitoring intervals, which can be found in Hsieh and Neuman (1985). For isotropic media, $\beta_{1}=2 R / B$ and $\beta_{2}=\cos \theta_{2}$, where $\theta_{2}$ is is the angle between the monitoring interval and the line connecting the centroids of the injection and monitoring intervals (Figure 1).

Then, Equation 12 gives the following expressions to infer the hydraulic conductivity and specific storage of an anisotropic formation,

$$
\sqrt{\frac{D}{K_{\mathrm{d}}(\mathbf{e})}}=\frac{Q s_{\mathrm{d}_{\mathrm{PL}}}(\infty)}{4 \pi R s^{*}}, \quad S_{\mathrm{s}}=\frac{\pi K_{\mathrm{d}}(\mathbf{e}) s_{\mathrm{dPL}}^{2}(\infty) t^{*}}{R^{2}}
$$

For isotropic formations, these reduce to

$$
K=\frac{Q s_{\mathrm{d}_{\mathrm{PL}}}(\infty)}{4 \pi R s^{*}}, \quad S_{s}=\frac{\pi K t^{*} s_{\mathrm{d}}^{2}(\infty)}{R^{2}}
$$

This approach should be used for data collected at

$$
t_{\mathrm{d}} \gg \frac{1-\beta_{2}^{2}}{4}, \quad t_{\mathrm{d}} \gg \frac{\left(\beta_{2} \pm 1 / \beta_{1}\right)^{2}}{4}
$$


Line Injection/Line Observation

The coefficients $A$ and $B$ in Equation 1 are given by Illman and Tartakovsky (2005b)

$$
A=\frac{Q s_{\mathrm{dLL}}(\infty)}{4 \pi L} \sqrt{\frac{K_{\mathrm{d}}\left(\mathbf{e}_{\mathrm{l}}\right)}{D}}, \quad B=\frac{Q R \mathrm{II}}{8 \pi L \alpha_{1}} \sqrt{\frac{K_{\mathrm{d}}\left(\mathbf{e}_{1}\right) S_{s}}{K_{\mathrm{d}}(\mathbf{e}) D}}
$$

where $s_{\mathrm{dLL}}(\infty)$ with $c \neq 1$ is given by equation 54 of Hsieh and Neuman (1985),

$$
\left.\begin{array}{l}
s_{\mathrm{dLL}}(\infty)=\frac{1}{2} \int_{-1}^{1} \ln \\
\times\left[\frac{\left\{\begin{array}{c}
\sqrt{\frac{\alpha_{1}^{2} \lambda^{2}}{\beta_{1}^{2}}+2 \lambda \frac{\alpha_{1}^{2} \beta_{2}+\alpha_{1} c}{\beta_{1}}+\alpha_{1}^{2}+2 \alpha_{1} \alpha_{2}+1} \\
+\alpha_{1} \alpha_{2}+1+\frac{\alpha_{1} c \lambda}{\beta_{1}}
\end{array}\right\}}{\left\{\begin{array}{l}
\sqrt{\frac{\alpha_{1}^{2} \lambda^{2}}{\beta_{1}^{2}}+2 \lambda \frac{\alpha_{1}^{2} \beta_{2}-\alpha_{1} c}{\beta_{1}}+\alpha_{1}^{2}-2 \alpha_{1} \alpha_{2}+1} \\
+\alpha_{1} \alpha_{2}-1+\frac{\alpha_{1} c \lambda}{\beta_{1}}
\end{array}\right)}\right] \mathrm{d}
\end{array}\right]
$$

$$
\begin{aligned}
\mathbb{I}= & \frac{\beta_{1}}{\sqrt{1-c^{2}}} \mathrm{e}^{C^{2}}\left[\operatorname{erf}\left(C+\frac{\sqrt{1-c^{2}}}{\beta_{1}}\right)\right. \\
& \left.-\operatorname{erf}\left(C-\frac{\sqrt{1-c^{2}}}{\beta_{1}}\right)\right]
\end{aligned}
$$

$C=\left(\beta_{2}-\alpha_{2} c\right) / \sqrt{1-c^{2}}$, and parameters $\alpha_{1} \geq 0,0 \leq \alpha_{2} \leq$ $1, \beta_{1} \geq 0$, and $0 \leq \beta_{2} \leq 1$, describing the geometric relationships between the injection and monitoring intervals, can be found in Hsieh and Neuman (1985). For isotropic media, $\alpha_{1}=2 R / L, \alpha_{2}=\cos \theta_{1}, \beta_{1}=2 R / B$, and $\beta_{2}=\cos$ $\theta_{2}$ (Figure 1). Note that $c$ has a meaning similar to $\alpha_{2}$ and $\beta_{2}$, and is related to the angle between the injection and monitoring intervals.

Then, Equation 17 gives the following expressions to infer the hydraulic conductivity and specific storage of an anisotropic formation,

$$
\sqrt{\frac{D}{K_{\mathrm{d}}\left(\mathbf{e}_{1}\right)}}=\frac{Q s_{\mathrm{dLL}}(\infty)}{4 \pi L s^{*}}, \quad S_{s}=\frac{4 \alpha_{1}^{2}}{R^{2} \mathbb{I}^{2}} s_{\mathrm{dLL}}^{2}(\infty) K_{\mathrm{d}}(\mathbf{e}) t^{*}
$$

For isotropic formations, these reduce to

$$
K=\frac{Q s_{\mathrm{dLL}}(\infty)}{4 \pi L s^{*}}, \quad S_{\mathrm{s}}=\frac{4 \alpha_{1}^{2} K t^{*}}{R^{2} \mathbb{I}^{2}} s_{\mathrm{dLL}}^{2}(\infty)
$$

This approach should be used for data collected at

$$
t_{\mathrm{d}} \gg \frac{1-\alpha_{2}^{2}}{4}, \quad t_{\mathrm{d}} \gg \frac{\left(\alpha_{2} \pm 1 / \alpha_{1}+\lambda c / \beta_{1}\right)^{2}}{4}
$$

\section{Application to a Cross-Hole Hydraulic Test in Fractured Granite}

We apply our technique to a cross-hole hydraulic test conducted in fractured granite around the Full-Scale
Engineered Barrier Experiment (FEBEX) gallery at the GTS in Switzerland.

\section{Site and Test Description}

The GTS, located in the Swiss Alps at an elevation of $1730 \mathrm{~m}$ above sea level, was selected to conduct research and development in the area of radioactive waste disposal. To support these objectives, various hydraulic tests were conducted within the FEBEX gallery, including pulse tests, single-hole hydraulic tests, cross-hole hydraulic tests, and tunnel inflow measurements.

The cross-hole tests conducted in the FEBEX gallery consist of four boreholes (between 70 and $150 \mathrm{~m}$ long) drilled from the main tunnel around the experimental site, and 19 boreholes (averaging $15 \mathrm{~m}$ in length) drilled according to a radial distribution from the gallery (Figure 2). The pumping and observation boreholes were divided into intervals of various lengths using 1-m-long inflatable packers resulting in 64 observation intervals. Figure 2 displays a scheme of each interval, which contains a pressure transducer, a water extraction/injection line for sampling purposes, and a water inlet to pressurize the packer itself. A detailed description of these experiments can be found in Martínez-Landa and Carrera (2005).

The test we analyzed involved pumping at a $12-\mathrm{m}$ long packed-off interval FBX2-4 (Figure 2), while observation took place in 64 intervals ranging between 0.80 and $40.0 \mathrm{~m}$ with an average length of $5.71 \mathrm{~m}$.

\section{Results}

The asymptotic analysis was conducted only on drawdown data that revealed a straight line at late time. To identify the correct model for data interpretation, we compared the length of the pumped interval with the lengths of the observation intervals and the radial distances between them. This comparison revealed that the pumped and observation intervals can be treated as a line and points, respectively, so that the asymptotic solution for the line-injection/point-observation flow configuration should be used.

The following simplifications might be used when warranted. The line-injection/point-observation solution reduces to the Theis (1935) solution in the limit of $\alpha_{1}$, $\alpha_{2} \rightarrow 0$ (Hsieh and Neuman 1985, equation 48). This occurs if the injection interval is considerably longer than the radial distance between the centroids of the injection and observation intervals. If the radial distance between the injection and observation intervals is much larger than the injection interval length, then the point-injection/ point-observation solution can be used in lieu of the lineinjection/point-observation solution. The theoretical limit of $\alpha_{1} \rightarrow \infty$ can be effectively employed when $\alpha_{1} \geq 5$ (Hsieh and Neuman 1985).

We found that all but two intervals used in the GTS cross-hole hydraulic test correspond to $\alpha_{1}$ within the range $0.2 \leq \alpha_{1} \leq 5.0$. The other two intervals had $\alpha_{1}>$ 5.0. This unambiguously suggests that, in the context of the GTS test, the Theis (1935) solution is a poor approximation of the line-injection/point-observation solution. 


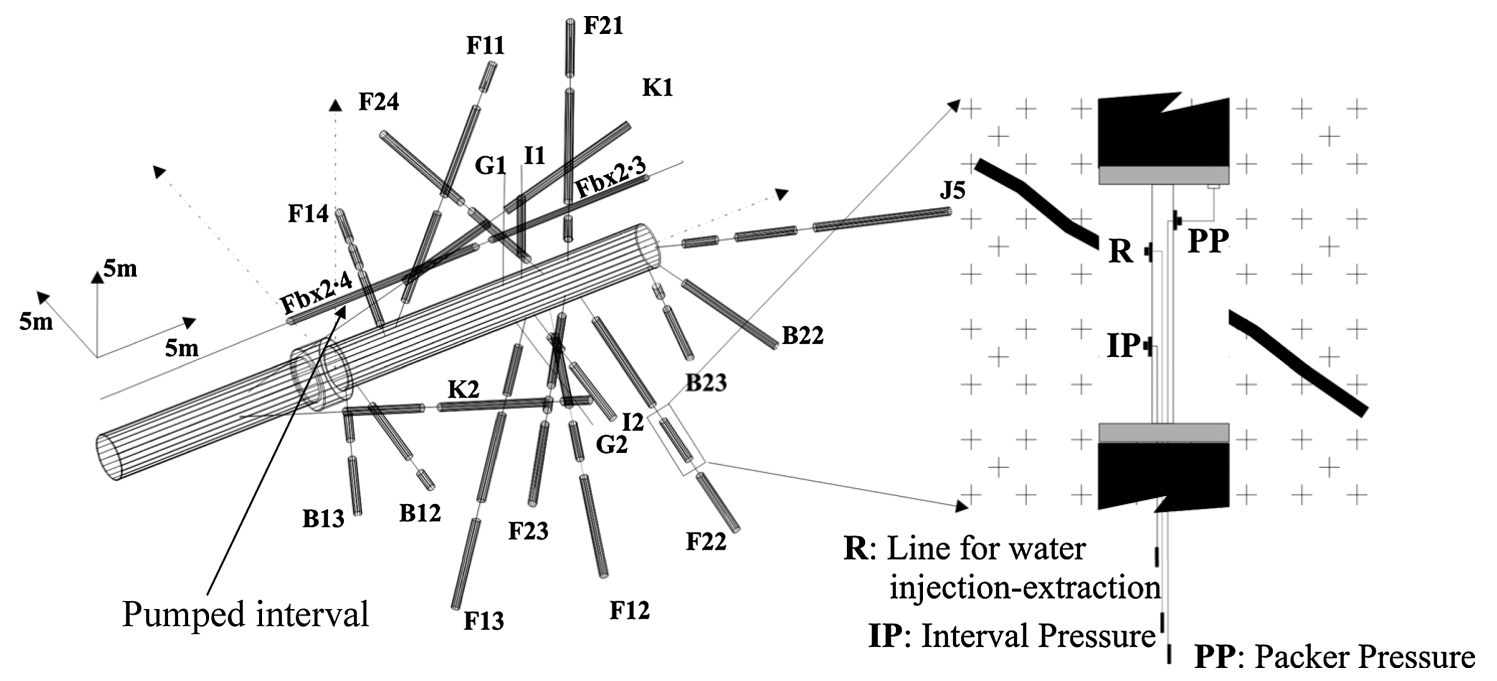

Figure 2. View of the 23 control boreholes in the test area. They are divided into intervals by means of inflatable packers. Each interval is equipped with a pressure intake, which is connected to an outer pressure transducer, and with a water recirculation intake for performing hydrochemical sampling and hydraulic tests. This leads to a total of 64 observation points (modified after Martínez-Landa and Carrera 2005).

Figure 3 shows drawdown data from interval B13-2 located $5.16 \mathrm{~m}$ away from the pumping interval. The inset figure depicts the double logarithm plot of the same data set. Both plots reveal a double-porosity effect, which we do not attempt to analyze due to lack of a full electronic record of the pumping rate. A visual examination of this and other drawdown records demonstrated that many of them attain a straight-line behavior at sufficiently large time and therefore are amenable to our asymptotic analysis.

The drawdown data in Figure 3 are representative of many drawdown records at the site, in that they exhibit a large signal-to-noise ratio. Hence, in principle, one can analyze these data not only with our asymptotic approach but with type-curve methods as well. The analysis of data from the remaining test intervals, including the drawdown record from interval F22-2 located $17.56 \mathrm{~m}$ away from the pumped interval (Figure 4), is much more challenging due to relatively small signal-to-noise ratios. In a typical fashion, the early data (large $t_{\mathrm{d}}{ }^{-1 / 2}$ ) in Figure 4 are

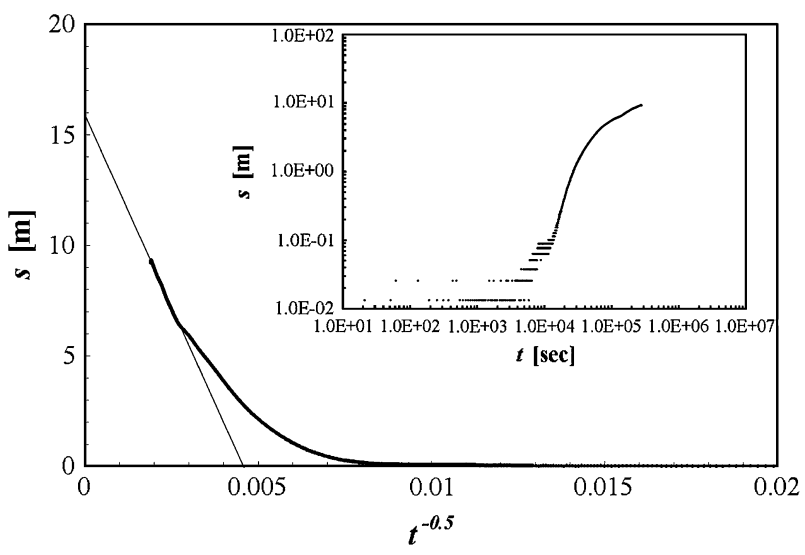

Figure 3. Asymptotic analysis of drawdown data from observation interval B13-2, with inset showing the doublelogarithmic plot. affected by significant noise, which renders the use of type-curve approaches problematic. However, the data become less noisy and a straight line develops at large times (small $t_{\mathrm{d}}{ }^{-1 / 2}$ ), which makes such data records amenable to our asymptotic analysis.

The estimates of $\log _{10}$-transformed hydraulic conductivity obtained with our asymptotic analysis from all monitoring interval data range from $-11.51(3.13 \times$ $\left.10^{-12} \mathrm{~m} / \mathrm{s}\right)$ to $-8.76\left(1.75 \times 10^{-9} \mathrm{~m} / \mathrm{s}\right)$ with a mean of $-9.86\left(1.39 \times 10^{-10} \mathrm{~m} / \mathrm{s}\right)$ and variance of 0.34 . The estimates of the $\log _{10^{-}}$-transformed specific storage range from $-8.83\left(1.49 \times 10^{-9} \mathrm{~m}^{-1}\right)$ to $-4.84\left(1.44 \times 10^{-5}\right.$ $\left.\mathrm{m}^{-1}\right)$ with a mean of $-6.06\left(8.76 \times 10^{-7} \mathrm{~m}^{-1}\right)$ and variance of 0.63 .

\section{Discussion}

We compare our estimates of hydraulic conductivity and specific storage with those derived from the same cross-hole tests by means of the Theis (1935) solution (Martínez-Landa and Jordan 2004; Martínez-Landa and Carrera 2005), pulse, recovery, and tunnel inflow tests. Cross-hole estimates of hydraulic conductivity and specific storage are also available from other tests conducted at the site.

The type-curve analysis of the 11 pumping and observation pairs (Martínez-Landa and Carrera 2005) revealed that $\log _{10}$-transformed hydraulic conductivity ranges from $-9.84\left(1.43 \times 10^{-10} \mathrm{~m} / \mathrm{s}\right)$ to $-8.64(2.31 \times$ $\left.10^{-9} \mathrm{~m} / \mathrm{s}\right)$ with a mean of $-9.49\left(3.22 \times 10^{-10} \mathrm{~m} / \mathrm{s}\right)$ and variance of 0.13 . The $\log _{10^{-}}$-transformed specific storage values range from $-7.25\left(5.68 \times 10^{-8} \mathrm{~m}^{-1}\right)$ to -5.76 $\left(1.74 \times 10^{-6} \mathrm{~m}^{-1}\right)$ with a mean of $-6.53\left(2.92 \times 10^{-7}\right.$ $\left.\mathrm{m}^{-1}\right)$ and variance of 0.33 .

Asymptotic analysis estimates for the same 11 pumping and observation pairs revealed that $\log _{10}$-transformed hydraulic conductivity $(K)$ ranges from $-11.51(3.13 \times$ 


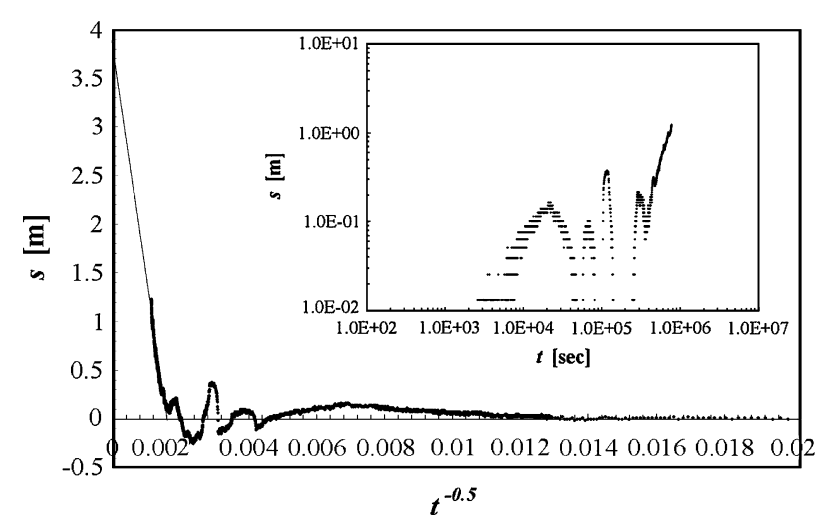

Figure 4. Asymptotic analysis of drawdown data from observation interval F22-2, with inset showing the doublelogarithmic plot.

$\left.10^{-12} \mathrm{~m} / \mathrm{s}\right)$ to $-10.02\left(9.60 \times 10^{-11} \mathrm{~m} / \mathrm{s}\right)$ with a mean of $-10.52\left(3.00 \times 10^{-11} \mathrm{~m} / \mathrm{s}\right)$ and variance of 0.19 . The $\log _{10}$-transformed specific storage $\left(S_{\mathrm{s}}\right)$ values range from $-8.83\left(1.49 \times 10^{-9} \mathrm{~m}^{-1}\right)$ to $-6.25\left(5.67 \times 10^{-7} \mathrm{~m}^{-1}\right)$ with a mean of $-6.93\left(1.17 \times 10^{-7} \mathrm{~m}^{-1}\right)$ and variance of 0.48 .

Thus, our estimates of both hydraulic conductivity and specific storage are smaller than their counterparts derived from the type-curve approach based on the Theis (1935) solution. This might be due to the fact that the conditions for the validity of the Theis approximation of crosshole test solutions discussed earlier have not been met.

Next, we compare our estimates of hydraulic conductivities with those obtained by other experimental techniques, i.e., pulse, recovery, and tunnel inflow measurements. Added to this comparison are the results of other cross-hole tests conducted at the site. The test type, number of measurements, mean, and variance for $\log _{10} K$ are summarized in Table 1. In general, our cross-hole results are in good agreement with the cross-hole estimates obtained by Martínez-Landa and Carrera (2005).
Martínez-Landa and Carrera (2005) found that, regardless of the type of hydraulic tests (pulse, recovery, cross-hole, and tunnel inflow measurements), the conceptualization of the fractured medium as a homogeneous continuum yields a strong scale effect, when the data are separated for intervals primarily measuring the matrix and fracture properties. For example, the mean of $\log _{10} K$ obtained through the pulse tests conducted in intervals, which the authors thought to be matrix dominated, is considerably smaller $(-10.70)$ than the mean $\log _{10} K$ from cross-hole tests $(-9.23$ in matrix intervals and -9.29 in fracture intervals). The important conclusions obtained by these authors are that (1) the scale effect is apparent and (2) the data interpretation with a numerical inverse model explicitly accounting for the conductive fractures in an otherwise homogeneous block of rock suppresses the scale effect. In particular, these authors found that the transmissivity values used in the model to represent fractures are consistent with those derived from both cross-hole tests and a few single-hole tests conducted at intervals intersecting dominant fractures. On the other hand, matrix hydraulic conductivities are consistent with the remaining single-hole tests. In summary, the majority of small-scale tests are performed in intervals dominated by the rock matrix. Thus, any averaging of these values would suggest relatively small effective hydraulic conductivity. Yet, large-scale permeability of the rock is controlled by a few fractures, which provide high connectivity to the system, but are intersected by a few testing intervals. As a result, large-scale hydraulic conductivity is qualitatively different and quantitatively larger than small-scale hydraulic conductivity (MartínezLanda and Carrera 2005).

A separate study involving single- and cross-hole pneumatic injection test data in unsaturated fractured tuff at the Apache Leop Research Site near Superior, Arizona, USA (Illman and Neuman 2001, 2003; Vesselinov et al. 2001; Hyun et al. 2002) also revealed a strong permeability scale effect. However, this finding is somewhat

\begin{tabular}{|c|c|c|c|c|}
\hline \multicolumn{5}{|c|}{$\begin{array}{c}\text { Table } 1 \\
\text { Sample Statistics of } \log _{10} K \text { from Analytic Interpretation of Pulse, Recovery, } \\
\text { Cross-Hole, and Tunnel Inflow Measurements }\end{array}$} \\
\hline Test Type & $n$ & Mean & $K(\mathbf{m} / \mathbf{s})$ & Variance \\
\hline Pulse (matrix) ${ }^{1}$ & 25 & -10.70 & $2.00 \mathrm{E}-11$ & 0.64 \\
\hline Pulse (fracture) ${ }^{1}$ & 8 & -7.79 & $1.62 \mathrm{E}-08$ & 0.36 \\
\hline Cross-hole (matrix) ${ }^{1}$ & 15 & -9.23 & $5.89 \mathrm{E}-10$ & 0.37 \\
\hline Cross-hole (fracture) ${ }^{1}$ & 14 & -9.29 & $5.13 \mathrm{E}-10$ & 0.39 \\
\hline Pulse $^{2}$ & 33 & -9.99 & $1.02 \mathrm{E}-10$ & 2.16 \\
\hline Recovery $^{2}$ & 21 & -9.75 & $1.78 \mathrm{E}-10$ & 1.51 \\
\hline Cross-hole ${ }^{2}$ & 29 & -9.26 & $5.50 \mathrm{E}-10$ & 0.37 \\
\hline Tunnel inflow ${ }^{2}$ & 4 & -9.47 & $3.39 \mathrm{E}-10$ & 0.00 \\
\hline Cross-hole ${ }^{3}$ & 40 & -9.86 & $1.38 \mathrm{E}-10$ & 0.34 \\
\hline \multicolumn{5}{|c|}{$\begin{array}{l}\text { Note: The terms "matrix" and "fracture" refer to intervals that are sparsely and heavily fractured, respectively. The statistics corresponding to "cross-hole (matrix)" } \\
\text { and "cross-hole (fracture)" test types represent data from the observation intervals. } \\
\text { 'The separate data analysis by Martínez-Landa and Carrera ( } 2005) \text {. } \\
{ }^{2} \text { The joint data analysis by Martínez-Landa and Carrera (2005). } \\
\text { 'The parameters inferred from our asymptotic analysis. }\end{array}$} \\
\hline
\end{tabular}


undermined by the use of different test types (single- and cross-hole tests), which results in different flow configurations and might introduce experimental and interpretive bias (e.g., Hsieh 1998; Zlotnik et al. 2000). This bias can be eliminated, or significantly reduced, if one relies on a single type of test conducted over a large range of scales. Illman (2006) employed this approach to analyze the data from cross-hole pneumatic injection tests, conducted in a tomographic manner at the ALRS, and found strong evidence of the directional permeability scale effect.

We use a similar strategy here to analyze the crosshole hydraulic tests in fractured granite at the GTS. Figures 5 and 6 exhibit log-log plots of the estimates of hydraulic conductivity and specific storage, respectively, as functions of the radial distance $R$ between the centroids of injection and observation intervals. The radial distance represents the nominal scale of the equivalent hydraulic parameters, and it ranges between 2.83 and $33.87 \mathrm{~m}$ with an arithmetic mean of $13.91 \mathrm{~m}$. The solid lines represent a linear model fit. Despite the moderate scatter in the data, both equivalent hydraulic parameters increase with the measurement scale, suggesting a scale effect of 3 orders of magnitude, which is similar in magnitude to that observed by Martínez-Landa and Carrera (2005, figure 9). Illman (2006) attributed the scatter of data to the strongly heterogeneous nature of the fractured rock at the ALRS. A similar argument can be made for the scatter at the GTS.

The existence and cause of the permeability scale effect are still a matter of considerable debate. The scale effect has been deemed illusory and variously attributed to such artifacts as (1) formation of skin around injection boreholes causing permeabilities obtained at the injection interval to be smaller than those determined in monitoring intervals (Butler and Healey 1998a, 1998b); (2) interpretation errors arising from the use of inconsistent theory (Zlotnik et al. 2000); (3) the dual nature of fractured rocks (Schulze-Makuch and Cherkauer 1998); (4) the use of data obtained by different measurement techniques (Hsieh 1998; Zlotnik et al. 2000; Martínez-Landa and Carrera 2005); (5) the use of data from tests conducted over several formations (Rovey and Cherkauer 1995); (6) directional effects (Neuman and Di Federico 2003);

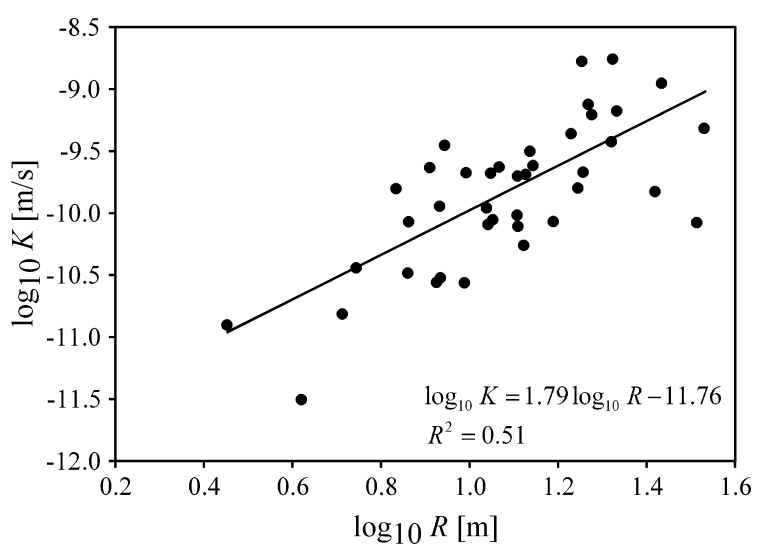

Figure 5. $\log _{10} K$ vs. $\log _{10} R$ with a linear model fit to the data set.

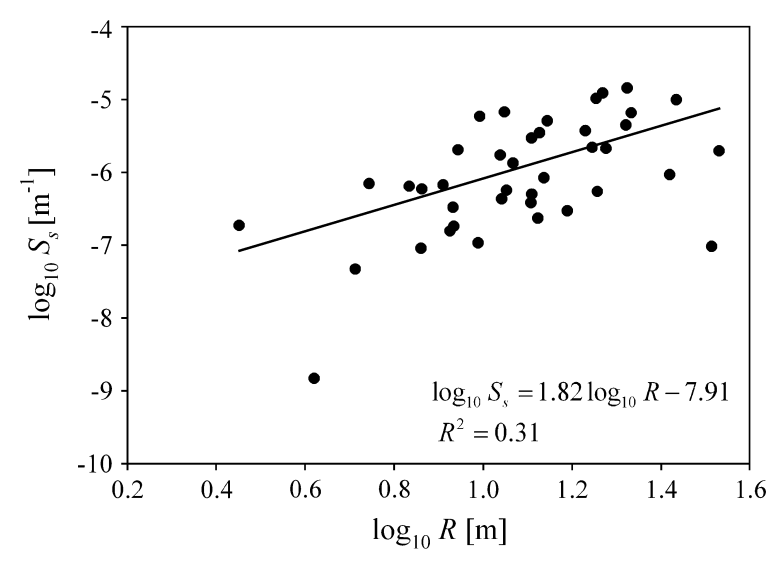

Figure 6. $\log _{10} S_{\mathrm{s}}$ vs. $\log _{10} R$ with a linear model fit to the data set.

and (7) the duration of pumping tests (Schulze-Makuch and Cherkauer 1998).

Hyun et al. (2002) and Illman (2006) argued that none of these apply to the cross-hole test data at the ALRS. Their reasoning is equally valid for the cross-hole test data at the GTS. It is reasonable to assume that the scale dependence of hydraulic parameters of fractured rocks stems from the connectivity of fluid-conducting fractures, whose scale variability changes with direction (Illman 2005, 2006). One can expect fracture connectivity to be a directional quantity since fractures tend to be oriented in the direction of minimum principal stress. This explains why permeability estimates obtained solely from single-hole tests do not increase significantly with the measurement scale because such tests measure the rock properties in the immediate vicinity of the injection interval where fracture connectivity is usually limited (Illman 2004). This additionally explains why permeability does not increase in a consistent fashion in all directions (Illman 2006) and, at other localities, might be nonexistent or limited (Hsieh 1998). Similar conclusions have been reached by Sanchez-Vila et al. (1996) and Knudby and Carrera (2005) through numerical simulations.

Finally, we plot $\log _{10} S_{\mathrm{s}}$ against $\log _{10} K$ in Figure 7 to study the correlation between these hydraulic parameters

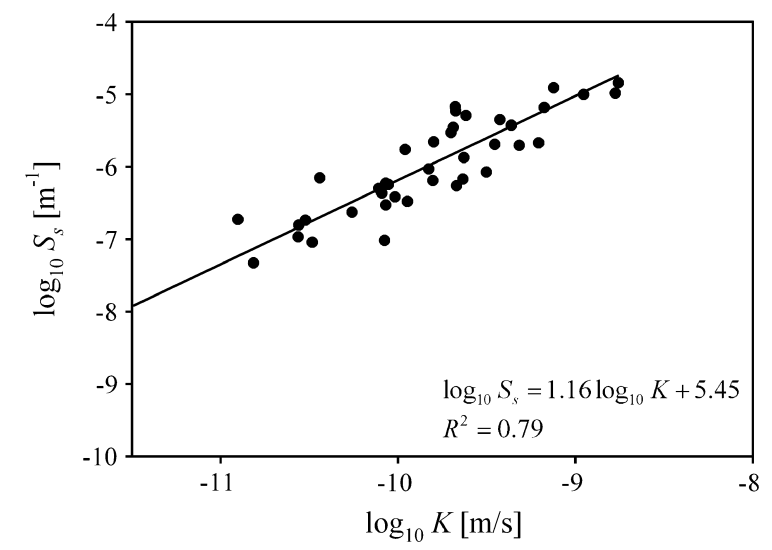

Figure 7. $\log _{10} S_{\mathrm{s}}$ vs. $\log _{10} K$ with a linear model fit to the data set. 
at the GTS. Fitting a straight line to these data by regression of $\log _{10} S_{S}$ on $\log _{10} K$ yields a high correlation between the two parameters with $r^{2}=0.79$. Similar correlations between conductive and storage properties of geologic media have been observed by Guimerà and Carrera (2000) and Vesselinov et al. (2001b).

\section{Conclusions}

Illman and Tartakovsky (2005a, 2005b) developed a new method to interpret three-dimensional pressure interference tests conducted in unsaturated porous or fractured geologic media based on the asymptotic analysis of late-time pressure-transient data. The approach yields reliable estimates of equivalent permeability and porosity through a simple straight-line fitting. We adopted this strategy to provide an asymptotic approach for the analysis of drawdown data collected from cross-hole hydraulic tests in aquifers and saturated fractured rocks. This approach was further employed to analyze data from the FEBEX, Grimsel, Switzerland. Our study leads to the following major conclusions:

(1) The proposed asymptotic approach provides a valuable alternative to (often highly subjective) type-curve analyses and (computationally intensive) numerical inverse models. It is applicable to data with low signal-to-noise ratios on which type-curve analyses often fail.

(2) Interpretation of cross-hole hydraulic tests conducted with variable injection and observation interval lengths requires a careful consideration of the well and interval geometries to determine the proper flow configuration, corresponding analytic solution, and its asymptotic representation. For the cross-hole hydraulic pumping test in fractured granite at the GTS, these are based on the lineinjection/point-observation solution of Hsieh and Neuman (1985).

(3) While our asymptotic analysis of the drawdown records treats the rock as hydraulically uniform and isotropic, it ultimately yields information about the spatial, scale, and directional dependence of the hydraulic connectivity, hydraulic conductivity, and specific storage of fractures across the site on scales relevant to the cross-hole test.

(4) The log-log plots of the estimates of hydraulic conductivity and specific storage vs. the radial distance between the centroids of the pumping and observation intervals revealed that both equivalent parameters increase with the measurement scale, suggesting a scale effect of 3 orders of magnitude, which is similar in magnitude to that observed by Martínez-Landa and Carrera (2005) through the comparison of small- (single-hole) and large-scale (cross-hole) hydraulic tests.

(5) The log-log plot of the estimates of specific storage vs. the estimates of hydraulic conductivity demonstrated a high correlation between these two hydraulic parameters in one of the hydraulic cross-hole tests at the GTS. Similar correlations between conductive and storage properties of geologic media have been observed by Guimerà and Carrera (2000) and Vesselinov et al. (2001b).

\section{Acknowledgments}

We thank Lurdes Martínez-Landa and Jesus Carrera for the GTS data and their assistance in data reduction. We also thank the two reviewers, Jesus Carrera and Frank Paillet, whose insightful comments helped us to improve the original version of the article. The first author was supported in part by funding from the National Science Foundation (NSF) and the Strategic Environmental Research \& Development Program (SERDP). The research by the second author was performed at Los Alamos National Laboratory under the auspices of the U.S. Department of Energy, contract W-7405-ENG-36, and was supported by the LDRD Program at Los Alamos National Laboratory.

\section{References}

Butler, J.J. Jr., and J.M. Healey. 1998a. Relationship between pumping-test and slug-test parameters: Scale effect or artifact? Ground Water 36, no. 2: 305-313.

Butler, J.J. Jr., and J.M. Healey. 1998b. Discussion of papers: Authors' reply. Ground Water 36, no. 6: 867-868.

Guimerà, J., and J. Carrera. 2000. A comparison of hydraulic and transport parameters measured in low permeability fractured media. Journal of Contaminant Hydrology 41, no. 3-4: 261-281.

Hsieh, P.A. 1998. Scale effects in fluid flow through fractured geologic media. In Scale Dependence and Scale Invariance in Hydrology, ed. G. Sposito, 335-353. Cambridge, UK: Cambridge University Press.

Hsieh, P.A., and S.P. Neuman. 1985. Field determination of the three-dimensional hydraulic conductivity tensor of anisotropic media, 1. Theory. Water Resources Research 21, no. 11: $1655-1665$.

Hsieh, P.A., S.P. Neuman, G.K. Stiles, and E.S. Simpson. 1985. Field determination of the three-dimensional hydraulic conductivity tensor of anisotropic media. 2. Methodology and application to fractured rocks.

Hyun, Y., S.P. Neuman, V.V. Vesselinov, W.A. Illman, D.M. Tartakovsky, and V. Di Federico. 2002. Theoretical interpretation of a pronounced permeability scale-effect in unsaturated fractured tuff. Water Resources Research 38, no. 6: 28-1-28-8.

Illman, W.A. 2006. Strong field evidence for directional permeability scale effect in fractured rock. Journal of Hydrology. In press.

Illman, W.A. 2005. Type curve analyses of pneumatic singlehole tests in unsaturated fractured tuff: Direct evidence for a porosity-scale effect. Water Resources Research 41, no. 4: W04018.

Illman, W.A. 2004. Analysis of permeability scaling within single boreholes. Geophysical Research Letters 31, no. 5: L06503.

Illman, W.A., and D.M. Tartakovsky. 2005a. Asymptotic analysis of three-dimensional pressure interference tests: Point source solution. Water Resources Research 41, no. 1: W01002.

Illman, W.A., and D.M. Tartakovsky. 2005b. Asymptotic analysis of cross-hole pneumatic injection tests in unsaturated fractured tuff. Advances in Water Resources 28, no. 11: 1217-1229.

Illman, W.A., and S.P. Neuman. 2003. Steady-state analyses of cross-hole pneumatic injection tests in unsaturated fractured tuff. Journal of Hydrology 281, no. 1-2: 36-54.

Illman, W.A., and S.P. Neuman. 2001. Type-curve interpretation of a cross-hole pneumatic test in unsaturated fractured tuff. Water Resources Research 37, no. 3: 583-604.

Illman, W.A., and S.P. Neuman. 2000. Type-curve interpretation of multi-rate single-hole pneumatic injection tests in unsaturated fractured rock. Ground Water 38, no. 6: 899-911. 
Knudby, C., and J. Carrera. 2005. On the relationship between indicators of geostatistical, flow and transport connectivity. Advances in Water Resources 28, no. 4: 405-421.

Martínez-Landa, L., and J. Carrera. 2005. An analysis of hydraulic conductivity scale effects in granite (Full-scale Engineered Barrier Experiment (FEBEX), Grimsel, Switzerland). Water Resources Research 41, no. 3: W03006.

Martínez-Landa L., and J. Jordan, 2004. Dismantling phase: 2003 Hydraulic Testing Campaign. Interpretation of short-duration and cross-hole tests. FEBEX project 70hyd-1-7-001. Barcelona, Spain: Technical University of Catalonia.

Neuman, S.P., and V. Di Federico. 2003. Multifaceted nature of hydrogeologic scaling and its interpretation. Reviews of Geophysics 41, no. 3: 1014.

Rovey, C.W. II, and D.S. Cherkauer. 1995. Scale dependency of hydraulic conductivity measurements. Ground Water 33, no. 5: 769-780.

Sánchez-Vila, X., J. Carrera, and J.P. Girardi. 1996. Scale effects in transmissivity. Journal of Hydrology 183, no. 1-2: $1-22$.
Schulze-Makuch, D., and D.S. Cherkauer. 1998. Variations in hydraulic conductivity with scale of measurements during aquifer tests in heterogeneous, porous carbonate rock. Hydrogeology Journal 6, no. 2: 204-215.

Theis, C.V. 1935. The relation between the lowering of the piezometric surface and rate and duration of discharge of a well using groundwater storage. Transactions of the American Geophysical Union 2, 519-524.

Vesselinov, V.V., S.P. Neuman, and W.A. Illman. 2001. Threedimensional numerical inversion of pneumatic cross-hole tests in unsaturated fractured tuff: 2. Equivalent parameters, high-resolution stochastic imaging and scale effects. Water Resources Research 37, no. 12: 3019-3042.

Zlotnik, V.A., B.R. Zurbuchen, T. Ptak, and G. Teutsch. 2000. Support volume and scale effect in hydraulic conductivity: Experimental aspects. In Theory, Modeling, and Field Investigation in Hydrogeology: A Special Volume in Honor of Shlomo P. Neuman's 60th Birthday, Geological Society of America Special Paper 348, eds. D. Zhang and C.L. Winter, 191-213. Boulder, Colorado: Geological Society of America.

\title{
Don't Let the Well Run Dry
}

You can make a difference in the life of others around the world in need of safe and plentiful supplies of ground water. Make your tax-deductible contribution today to the Developing World Fund of the National Ground Water Research and Educational Foundation.

Established in 1994, NGWREF is operated as a $501 \mathrm{c}(3)$ foundation by the National Ground Water Association and is focused on education, research, and other charitable activities related to a broader understanding of ground water.

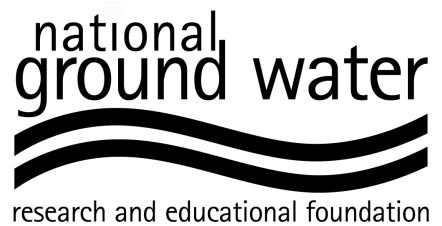

\author{
National Ground Water Research and Educational Foundation \\ 601 Dempsey Road \\ Westerville, Ohio 43081 \\ 800551.7379 \\ 800898.7786 fax
}

www.ngwa.org/ngwef/ngwef.html 